\title{
Studying High-Mass Microquasars with HAWC
}

\section{Chang Dong Rho, ${ }^{a, *}$ Ke Fang, ${ }^{b}$ Se Yeon Hwang ${ }^{c}$ and Youngwan Son ${ }^{c}$ on behalf of the HAWC Collaboration}

(a complete list of authors can be found at the end of the proceedings)

${ }^{a}$ University of Seoul, Natural Science Research Institute

Seoul, Republic of Korea

${ }^{b}$ University of Wisconsin-Madison, Department of Physics

Madison, WI, USA

${ }^{c}$ University of Seoul, Department of Physics

Seoul, Republic of Korea

E-mail: cdr397@uos.ac.kr

High-mass microquasars (HMMQs) are powerful particle accelerators, but their mechanism of the high-energy emission is poorly understood. To date, only a handful of these particle engines have ever been observed to emit gamma-ray photons and are thus potential $\mathrm{TeV}$ gamma-ray emitters. In this work, we study four HMMQs, namely, LS 5039, Cyg X-1, Cyg X-3, and SS 433 using the data from the High Altitude Water Cherenkov (HAWC) observatory. We perform time dependent analyses on each HMMQ to look for any periodic variations in their flux. We produce light curves using the HAWC daily maps from which we generate Lomb-Scargle periodograms. By analysing the significance of the periodogram peaks, we assess whether or not HAWC is sensitive to orbitally modulating TeV gamma-ray flux in the four HMMQs.

$37^{\text {th }}$ International Cosmic Ray Conference (ICRC 2021)

July 12th - 23rd, 2021

Online - Berlin, Germany

\footnotetext{
*Presenter
} 


\section{Introduction}

Gamma-ray binaries are sources that have non-thermal emission peaking above $1 \mathrm{MeV}$ in their spectra with orbital modulation in their gamma-ray flux [1]. Consequently, very-high-energy (VHE) gamma-ray binaries are those that exhibit periodic variation in their VHE gamma-ray flux and are extremely rare to find. Due to the limited number of observations and lack of a standard model, our understanding of the mechanisms that drive VHE gamma-ray binaries is still poor. One of the models that exists is high-mass microquasar (HMMQ) where the "high-mass" refers to the massive companion star. Microquasars are a type of compact binaries that have either a neutron star or black hole as their compact object and they have materials from their massive companion that accrete onto the compact object forming an accretion disk. Microquasars also have relativistic jets ejected to either side of the disk, a feature that is also seen in active galactic nuclei or quasars, only on smaller scales. The reasons for VHE gamma-ray flux modulation is also not clearly understood because the VHE gamma-ray binaries do not always follow the same pattern [1]. However, to briefly discuss some of the factors, anisotropy in Compton effect would cause higher flux when the photon electron collision is head on with respect to the observer. Secondly, assuming the accretion rate and the jets contribute to the production of gamma-ray emission, the wind accretion rate is inversely proportional to the relative velocity of the compact object, which could vary with orbital position. Lastly, maximum flux from VHE gamma-ray pair production absorption would occur when the gamma-photon interaction is head-on [1].

The results provided in this work follow up on "HAWC Search for High-mass Microquasars" [2], which presents TeV gamma-ray flux upper limits on the HMMQs within the HAWC field of view and discusses two scenarios that may explain potential VHE gamma-ray production. This proceeding extends on [2] by looking at the time-dependent aspect of the same HMMQs.

\section{HAWC}

The High Altitude Water Cherenkov (HAWC) Observatory is a ground based particle sampling array designed to indirectly observe gamma rays. In order to efficiently collect particles produced from gamma-ray air showers, HAWC is located at a high altitude of approximately $4.1 \mathrm{~km}$ between Sierra Negra and Pico de Orizaba in Puebla, Mexico. The main array of HAWC consists of 300 water Cherenkov detectors (WCDs) covering a combined geometrical area of $\sim 22,000 \mathrm{~m}^{2}$. Each WCD is $4.5 \mathrm{~m}$ tall with $7.3 \mathrm{~m}$ diameter and is filled with $\sim 200,000 \mathrm{~L}$ of purified water. At the bottom of each WCD, there are four photomultiplier tubes (PMTs) anchored so when charged daughter particles produced from air showers propagate through the WCD, the emitted Cherenkov light can be collected for indirect detection of gamma rays. Also, the WCDs are sufficiently deep enough to act as calorimeters as well as the detector medium. For each triggered event, HAWC uses the PMT charge spatial distribution to identify the "core" of the shower and uses the timing information to reconstruct the incidence angle of the original gamma-ray photon and thus the position of its source. The typical trigger rate of HAWC is approximately $25 \mathrm{kHz}$. However, most of the triggered events are cosmic ray background that are later filtered out using gamma-hadron separators [3]. The official energy range of HAWC is between $300 \mathrm{GeV}$ and $100 \mathrm{TeV}$. 
Some of the main advantages of HAWC are its high duty cycle (>95\%) and wide field of view (2 sr) [3]. These aspects allow HAWC to be suitable for carrying out time dependent analyses on binary systems.

The data used for the analysis presented in this proceeding are HAWC daily maps, each with one-transit (one day) worth of data, spanning from 2014 November 26th to 2020 October 7th.

\section{Results}

Here, we present the results. The first set are the daily light curves for the four HMMQs as shown in Figure 1. Each data point corresponds to the fit result on each HAWC daily map as described in Section 2.

Since the four HMMQs are all located in source confused regions and that the daily maps each have very small portions of the full HAWC data, studying light curves alone may not easily identify periodic orbital modulation in flux. Therefore, we applied the Lomb-Scargle periodogram $[4,5]$ to further analyse the light curves and their potential periodicities. Figure 2 shows the periodograms corresponding to the light curves (Figure 1). Here, the green dashed lines indicate each of their known periods: 5.6 days for Cyg X-1 [6]; 3.9 days for LS 5039 [7]; and 13.1 days for SS 433 [8]. The red dashed lines, on the other hand, indicate the highest peaks found from the periodograms.

The periodogram for Cyg X-3 is missing because the known period for Cyg X-3 is 0.2 days [9], which is shorter than the minimum time resolution of the HAWC data used (1 day), thus the periodogram analysis cannot be performed properly for Cyg X-3.

\section{Discussion}

Figure 2 shows that there is no significant peak at or close to the known periods of the HMMQs we are studying. As for the highest peaks, the significance of the peaks need to be computed to see if HAWC is actually observing the flux variations in HMMQs at these other periods because these peaks could simply be due to background fluctuations. To confirm, we created 10,000 pseudo light curves with background-only data for each HMMQ and made a periodogram for each light curve. Then, the power corresponding to the highest peak of each periodogram is collected to form a histogram. By looking at the power distribution on the histogram, we can compute the p-value for the original highest peak. The histograms are presented in Figure 2, which show that the three HMMQs studied had the maximum peak that had a significantly small $\mathrm{p}$ value.

As mentioned before, LS 5039 is the one HMMQ that has been detected via TeV gamma rays [10]. Through this detection, H.E.S.S. showed that this source has high and low states. Hard x-ray data also show that the HMMQs have high and low states. Assuming that there is correlation between the hard x-ray emission and VHE gamma-ray emission, collecting the parts of the HAWC data corresponding only to the high or hard states may provide the required data for HAWC to be sensitive enough to observe periodic emission. Hence, we plan to continue the analysis by concentrating on different parts of the data, more useful for each source. The Lomb-Scargle periodogram can analyse data with nonuniform time spacing, hence both time-dependent and timeindependent analysis can be performed on the high-state only data. On the other hand, the high 

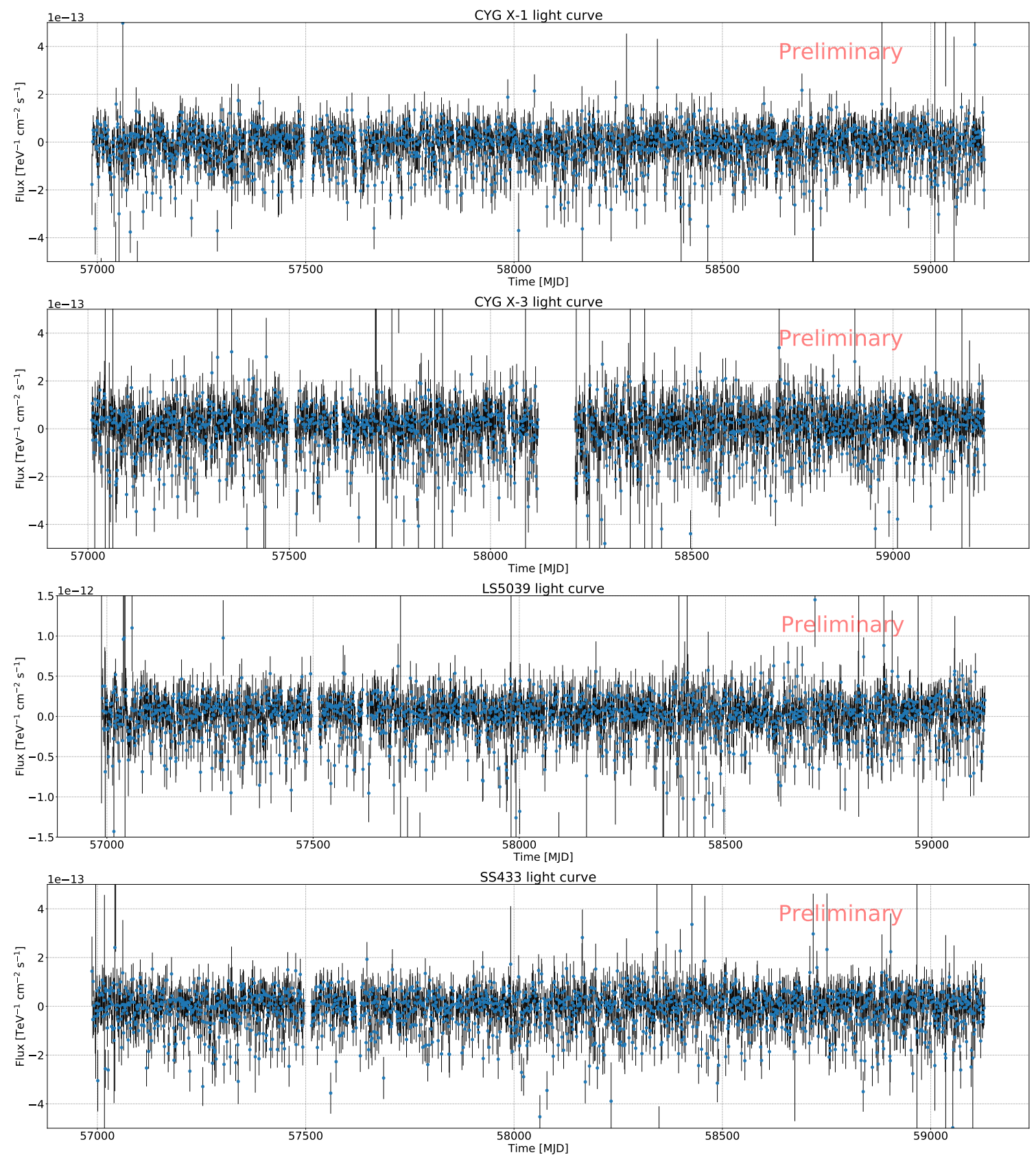

Figure 1: Daily light curves for the four HMMQs, from top to bottom: Cyg X-1; Cyg X-3; LS 5039; and SS 433, produced using HAWC data.

states of LS 5039 show a hard cut off above a few TeV [10]. Therefore, depending on the cut-off energy, HAWC may not be able to observe the HMMQs even with the high-state data.

Another problem is that the four HMMQs are located in source confused regions with Galactic diffuse emission contamination. Therefore, multi-source fitting is preferred. 

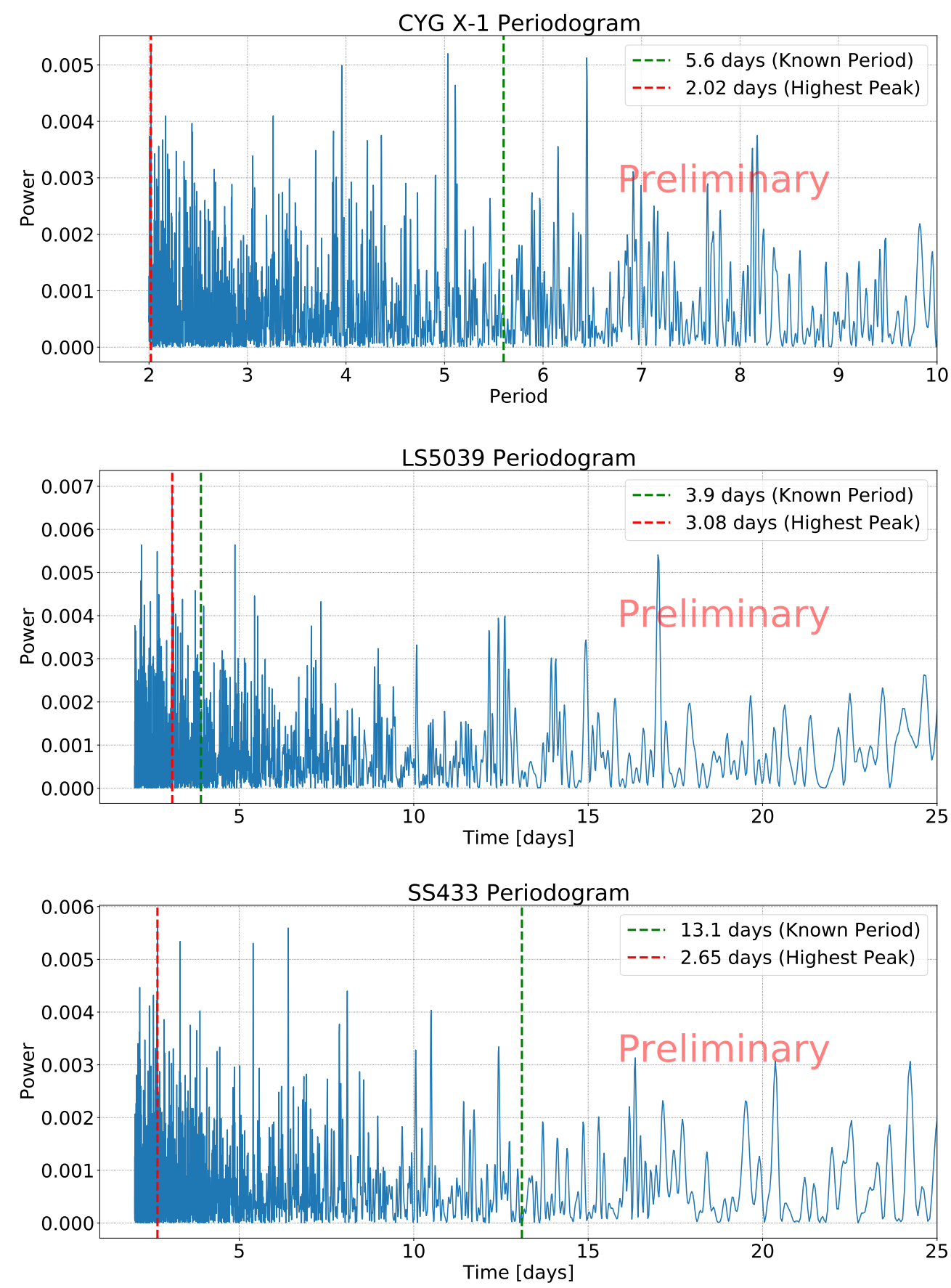

Figure 2: Lomb-Scargle periodograms generated from the light curves in Figure 1. From top to bottom, these are for Cyg X-1, LS 5039, and SS 433. The green dashed lines indicate the known orbital periods of the HMMQs. The red dashed lines are mark where the maximum periodogram peaks are found.

\section{Conclusion}

We have carried out a follow-up time dependent study on the four HMMQs, namely, LS 5039, Cyg X-1, Cyg X-3, and SS 433. We looked at their light curves and periodograms to look for any 

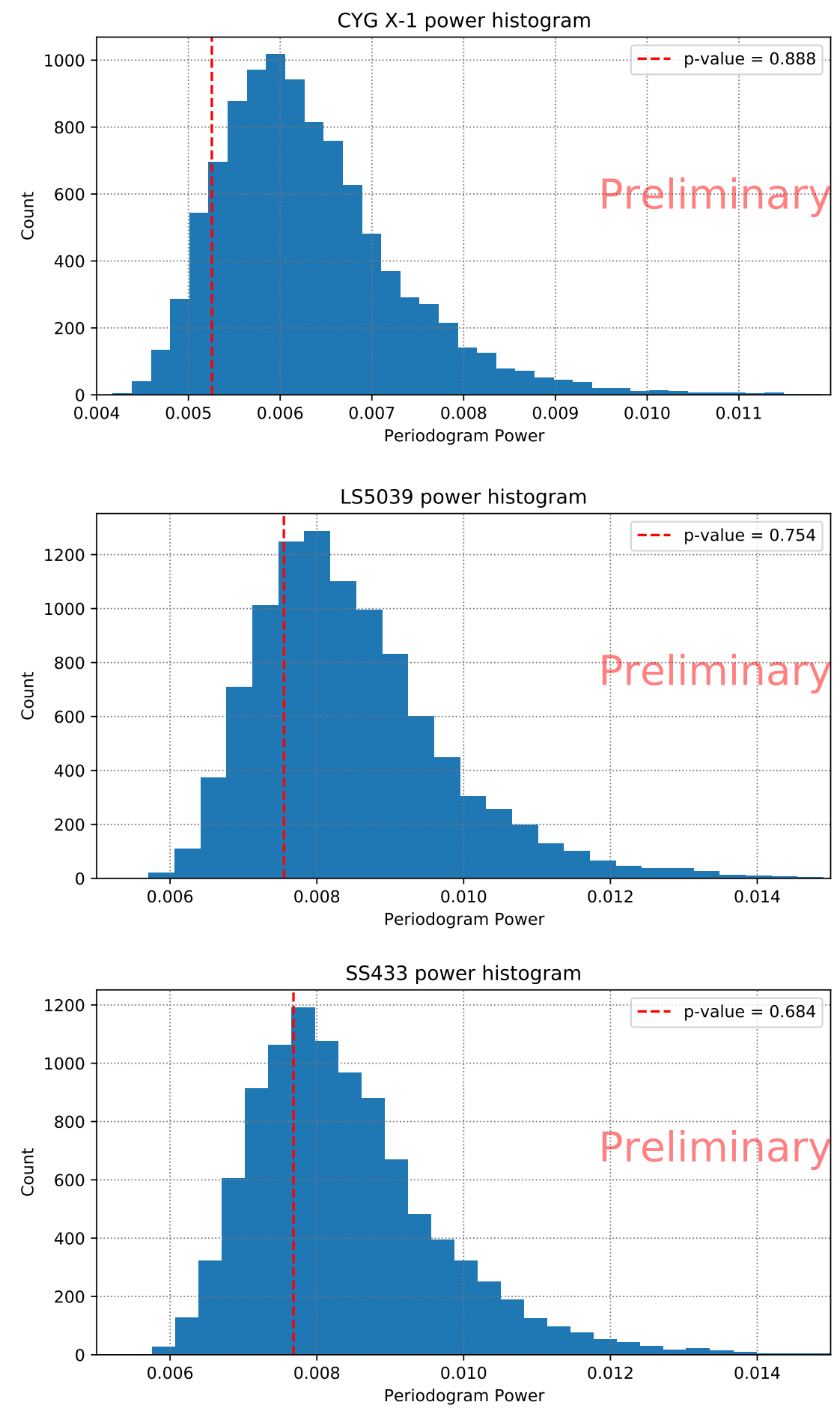

Figure 3: Peak power histograms produced from 10,000 pseudo-light-curves and their periodograms. The red dashed lines indicate the p-values corresponding to the peak power from the original periodograms (Figure 2). 
signs of periodically varying flux due to an orbital motion of each binary system. However, using one-day transit HAWC maps, no significant evidence could be found. We plan to continue studying the HMMQs by looking at only the part of the data that correspond to X-ray hard states and doing multi-source fits of the regions of interest.

\section{Acknowledgments}

We acknowledge the support from: the US National Science Foundation (NSF); the US Department of Energy Office of High-Energy Physics; the Laboratory Directed Research and Development (LDRD) program of Los Alamos National Laboratory; Consejo Nacional de Ciencia y Tecnología (CONACyT), México, grants 271051, 232656, 260378, 179588, 254964, 258865, 243290, 132197, A1-S-46288, A1-S-22784, cátedras 873, 1563, 341, 323, Red HAWC, México; DGAPA-UNAM grants IG101320, IN111716-3, IN111419, IA102019, IN110621, IN110521; VIEP-BUAP; PIFI 2012, 2013, PROFOCIE 2014, 2015; the University of Wisconsin Alumni Research Foundation; the Institute of Geophysics, Planetary Physics, and Signatures at Los Alamos National Laboratory; Polish Science Centre grant, DEC-2017/27/B/ST9/02272; Coordinación de la Investigación Científica de la Universidad Michoacana; Royal Society - Newton Advanced Fellowship 180385; Generalitat Valenciana, grant CIDEGENT/2018/034; Chulalongkorn University's CUniverse (CUAASC) grant; Coordinación General Académica e Innovación (CGAI-UdeG), PRODEP-SEP UDG-CA499; Institute of Cosmic Ray Research (ICRR), University of Tokyo, H.F. acknowledges support by NASA under award number 80GSFC21M0002. We also acknowledge the significant contributions over many years of Stefan Westerhoff, Gaurang Yodh and Arnulfo Zepeda Dominguez, all deceased members of the HAWC collaboration. Thanks to Scott Delay, Luciano Díaz and Eduardo Murrieta for technical support.

\section{References}

[1] G. Dubus (2013) AAR, 21, 64

[2] A. Albert et al. (2021) ApJL 912 L4

[3] A. U. Abeysekara et al. (2017) ApJ 84339

[4] N. R. Lomb (1976) Ap\&SS 39447

[5] J. D. Scargle (1982) ApJ 263835

[6] C. Brocksopp et al. (1999) A\&A 343: 861-864

[7] J. Casares et al. (2005) MNRAS 364 (3): 899-908

[8] B. Margon (1984) ARA\&A 22 (1): 507-536

[9] C. Canizares et al. (1973) Nat. Phys. Sci. 241, 28-30

[10] C. Mariaud et al. (2016) PoS ICRC2015 885 


\section{Full Authors List: HAWC Collaboration}

A.U. Abeysekara ${ }^{48}$, A. Albert ${ }^{21}$, R. Alfaro ${ }^{14}$, C. Alvarez ${ }^{41}$, J.D. Álvarez ${ }^{40}$, J.R. Angeles Camacho ${ }^{14}$, J.C. Arteaga-Velázquez ${ }^{40}$, K. P. Arunbabu $^{17}$, D. Avila Rojas ${ }^{14}$, H.A. Ayala Solares ${ }^{28}$, R. Babu ${ }^{25}$, V. Baghmanyan ${ }^{15}$, A.S. Barber ${ }^{48}$, J. Becerra Gonzalez ${ }^{11}$, E. BelmontMoreno $^{14}$, S.Y. BenZvi ${ }^{29}$, D. Berley ${ }^{39}$, C. Brisbois ${ }^{39}$, K.S. Caballero-Mora ${ }^{41}$, T. Capistrán ${ }^{12}$, A. Carramiñana ${ }^{18}$, S. Casanova ${ }^{15}$, O. Chaparro-Amaro $^{3}$, U. Cotti ${ }^{40}$, J. Cotzomi ${ }^{8}$, S. Coutiño de León ${ }^{18}$, E. De la Fuente ${ }^{46}$, C. de León ${ }^{40}$, L. Diaz-Cruz ${ }^{8}$, R. Diaz Hernandez ${ }^{18}$, J.C. Díaz-Vélez ${ }^{46}$, B.L. Dingus ${ }^{21}$, M. Durocher ${ }^{21}$, M.A. DuVernois ${ }^{45}$, R.W. Ellsworth ${ }^{39}$, K. Engel ${ }^{39}$, C. Espinoza ${ }^{14}$, K.L. Fan ${ }^{39}$, K. Fang $^{45}$, M. Fernández Alonso ${ }^{28}$, B. Fick ${ }^{25}$, H. Fleischhack ${ }^{51,11,52}$, J.L. Flores ${ }^{46}$, N.I. Fraija ${ }^{12}$, D. Garcia ${ }^{4}$, J.A. García-González ${ }^{20}$, J. L. García-Luna ${ }^{46}$, G. García-Torales ${ }^{46}$, F. Garfias ${ }^{12}$, G. Giacinti ${ }^{22}$, H. Goksu ${ }^{22}$, M.M. González ${ }^{12}$, J.A. Goodman ${ }^{39}$, J.P. Harding ${ }^{21}$, S. Hernandez ${ }^{14}$, I. Herzog ${ }^{25}$, J. Hinton ${ }^{22}$, B. Hona ${ }^{48}$, D. Huang ${ }^{25}$, F. Hueyotl-Zahuantitla ${ }^{41}$, C.M. Hui ${ }^{23}$, B. Humensky ${ }^{39}$, P. Hüntemeyer ${ }^{25}$, A. Iriarte ${ }^{12}$, A. Jardin-Blicq ${ }^{22,49,50}$, H. Jhee ${ }^{43}$, V. Joshi ${ }^{7}$, D. Kieda ${ }^{48}$, G J. Kunde ${ }^{21}$, S. Kunwar ${ }^{22}$, A. Lara ${ }^{17}$, J. Lee ${ }^{43}$, W.H. Lee ${ }^{12}$, D. Lennarz ${ }^{9}$, H. León Vargas ${ }^{14}$, J. Linnemann ${ }^{24}$, A.L. Longinotti ${ }^{12}$, R. López-Coto ${ }^{19}$, G. Luis-Raya ${ }^{44}$, J. Lundeen ${ }^{24}$, K. Malone ${ }^{21}$, V. Marandon $^{22}$, O. Martinez ${ }^{8}$, I. Martinez-Castellanos ${ }^{39}$, H. Martínez-Huerta ${ }^{38}$, J. Martínez-Castro ${ }^{3}$, J.A.J. Matthews ${ }^{42}$, J. McEnery ${ }^{11}$, P. Miranda-Romagnoli $^{34}$, J.A. Morales-Soto ${ }^{40}$, E. Moreno ${ }^{8}$, M. Mostafá ${ }^{28}$, A. Nayerhoda ${ }^{15}$, L. Nellen ${ }^{13}$, M. Newbold ${ }^{48}$, M.U. Nisa ${ }^{24}$, R. Noriega-Papaqui ${ }^{34}$, L. Olivera-Nieto ${ }^{22}$, N. Omodei ${ }^{32}$, A. Peisker ${ }^{24}$, Y. Pérez Araujo ${ }^{12}$, E.G. Pérez-Pérez ${ }^{44}$, C.D. Rho ${ }^{43}$, C. Rivière ${ }^{39}$, D. Rosa-Gonzalez ${ }^{18}$, E. Ruiz-Velasco ${ }^{22}$, J. Ryan ${ }^{26}$, H. Salazar ${ }^{8}$, F. Salesa Greus ${ }^{15,53}$, A. Sandoval ${ }^{14}$, M. Schneider ${ }^{39}$, H. Schoorlemmer ${ }^{22}$, J. Serna-Franco ${ }^{14}$, G. Sinnis ${ }^{21}$, A.J. Smith ${ }^{39}$, R.W. Springer ${ }^{48}$, P. Surajbali ${ }^{22}$, I. Taboada ${ }^{9}$, M. Tanner ${ }^{28}$, K. Tollefson ${ }^{24}$, I. Torres ${ }^{18}$, R. Torres-Escobedo $^{30}$, R. Turner ${ }^{25}$, F. Ureña-Mena ${ }^{18}$, L. Villaseñor ${ }^{8}$, X. Wang ${ }^{25}$, I.J. Watson ${ }^{43}$, T. Weisgarber ${ }^{45}$, F. Werner ${ }^{22}$, E. Willox ${ }^{39}$, J. Wood ${ }^{23}$, G.B. Yodh ${ }^{35}$, A. Zepeda ${ }^{4}$, H. Zhou ${ }^{30}$

${ }^{1}$ Barnard College, New York, NY, USA, ${ }^{2}$ Department of Chemistry and Physics, California University of Pennsylvania, California, PA, USA, ${ }^{3}$ Centro de Investigación en Computación, Instituto Politécnico Nacional, Ciudad de México, México, ${ }^{4}$ Physics Department, Centro de Investigación y de Estudios Avanzados del IPN, Ciudad de México, México, ${ }^{5}$ Colorado State University, Physics Dept., Fort Collins, CO, USA, ${ }^{6}$ DCI-UDG, Leon, Gto, México, ${ }^{7}$ Erlangen Centre for Astroparticle Physics, Friedrich Alexander Universität, Erlangen, BY, Germany, ${ }^{8}$ Facultad de Ciencias Físico Matemáticas, Benemérita Universidad Autónoma de Puebla, Puebla, México, ${ }^{9}$ School of Physics and Center for Relativistic Astrophysics, Georgia Institute of Technology, Atlanta, GA, USA, ${ }^{10}$ School of Physics Astronomy and Computational Sciences, George Mason University, Fairfax, VA, USA, ${ }^{11}$ NASA Goddard Space Flight Center, Greenbelt, MD, USA, ${ }^{12}$ Instituto de Astronomía, Universidad Nacional Autónoma de México, Ciudad de México, México, ${ }^{13}$ Instituto de Ciencias Nucleares, Universidad Nacional Autónoma de México, Ciudad de México, México, ${ }^{14}$ Instituto de Física, Universidad Nacional Autónoma de México, Ciudad de México, México, ${ }^{15}$ Institute of Nuclear Physics, Polish Academy of Sciences, Krakow, Poland, ${ }^{16}$ Instituto de Física de São Carlos, Universidade de São Paulo, São Carlos, SP, Brasil, ${ }^{17}$ Instituto de Geofísica, Universidad Nacional Autónoma de México, Ciudad de México, México, ${ }^{18}$ Instituto Nacional de Astrofísica, Óptica y Electrónica, Tonantzintla, Puebla, México, ${ }^{19}$ INFN Padova, Padova, Italy, ${ }^{20}$ Tecnologico de Monterrey, Escuela de Ingeniería y Ciencias, Ave. Eugenio Garza Sada 2501, Monterrey, N.L., 64849, México, ${ }^{21}$ Physics Division, Los Alamos National Laboratory, Los Alamos, NM, USA, ${ }^{22}$ Max-Planck Institute for Nuclear Physics, Heidelberg, Germany, ${ }^{23}$ NASA Marshall Space Flight Center, Astrophysics Office, Huntsville, AL, USA, ${ }^{24}$ Department of Physics and Astronomy, Michigan State University, East Lansing, MI, USA, ${ }^{25}$ Department of Physics, Michigan Technological University, Houghton, MI, USA, ${ }^{26}$ Space Science Center, University of New Hampshire, Durham, NH, USA, ${ }^{27}$ The Ohio State University at Lima, Lima, OH, USA, ${ }^{28}$ Department of Physics, Pennsylvania State University, University Park, PA, USA, ${ }^{29}$ Department of Physics and Astronomy, University of Rochester, Rochester, NY, USA, ${ }^{30}$ Tsung-Dao Lee Institute and School of Physics and Astronomy, Shanghai Jiao Tong University, Shanghai, China, ${ }^{31}$ Sungkyunkwan University, Gyeonggi, Rep. of Korea, ${ }^{32}$ Stanford University, Stanford, CA, USA, ${ }^{33}$ Department of Physics and Astronomy, University of Alabama, Tuscaloosa, AL, USA, ${ }^{34}$ Universidad Autónoma del Estado de Hidalgo, Pachuca, Hgo., México, ${ }^{35}$ Department of Physics and Astronomy, University of California, Irvine, Irvine, CA, USA, ${ }^{36}$ Santa Cruz Institute for Particle Physics, University of California, Santa Cruz, Santa Cruz, CA, USA, ${ }^{37}$ Universidad de Costa Rica, San José, Costa Rica, ${ }^{38}$ Department of Physics and Mathematics, Universidad de Monterrey, San Pedro Garza García, N.L., México, ${ }^{39}$ Department of Physics, University of Maryland, College Park, MD, USA, ${ }^{40}$ Instituto de Física y Matemáticas, Universidad Michoacana de San Nicolás de Hidalgo, Morelia, Michoacán, México, ${ }^{41}$ FCFM-MCTP, Universidad Autónoma de Chiapas, Tuxtla Gutiérrez, Chiapas, México, ${ }^{42}$ Department of Physics and Astronomy, University of New Mexico, Albuquerque, NM, USA, ${ }^{43}$ University of Seoul, Seoul, Rep. of Korea, ${ }^{44}$ Universidad Politécnica de Pachuca, Pachuca, Hgo, México, ${ }^{45}$ Department of Physics, University of Wisconsin-Madison, Madison, WI, USA, ${ }^{46}$ CUCEI, CUCEA, Universidad de Guadalajara, Guadalajara, Jalisco, México, ${ }^{47}$ Universität Würzburg, Institute for Theoretical Physics and Astrophysics, Würzburg, Germany, ${ }^{48}$ Department of Physics and Astronomy, University of Utah, Salt Lake City, UT, USA, ${ }^{49}$ Department of Physics, Faculty of Science, Chulalongkorn University, Pathumwan, Bangkok 10330, Thailand, ${ }^{50}$ National Astronomical Research Institute of Thailand (Public Organization), Don Kaeo, MaeRim, Chiang Mai 50180, Thailand, ${ }^{51}$ Department of Physics, Catholic University of America, Washington, DC, USA, ${ }^{52}$ Center for Research and Exploration in Space Science and Technology, NASA/GSFC, Greenbelt, MD, USA, ${ }^{53}$ Instituto de Física Corpuscular, CSIC, Universitat de València, Paterna, Valencia, Spain 